TRANSACTIONS OF THE

AMERICAN MATHEMATICAL SOCIETY

Volume 357, Number 9, Pages 3605-3621

S 0002-9947(04)03591-3

Article electronically published on November 4, 2004

\title{
DEPTH AND COHOMOLOGICAL CONNECTIVITY IN MODULAR INVARIANT THEORY
}

\author{
PETER FLEISCHMANN, GREGOR KEMPER, AND R. JAMES SHANK
}

\begin{abstract}
Let $G$ be a finite group acting linearly on a finite-dimensional vector space $V$ over a field $K$ of characteristic $p$. Assume that $p$ divides the order of $G$ so that $V$ is a modular representation and let $P$ be a Sylow $p$-subgroup for $G$. Define the cohomological connectivity of the symmetric algebra $S\left(V^{*}\right)$ to be the smallest positive integer $m$ such that $H^{m}\left(G, S\left(V^{*}\right)\right) \neq 0$. We show that $\min \left\{\operatorname{dim}_{K}\left(V^{P}\right)+m+1, \operatorname{dim}_{K}(V)\right\}$ is a lower bound for the depth of $S\left(V^{*}\right)^{G}$. We characterize those representations for which the lower bound is sharp and give several examples of representations satisfying the criterion. In particular, we show that if $G$ is $p$-nilpotent and $P$ is cyclic, then, for any modular representation, the depth of $S\left(V^{*}\right)^{G}$ is $\min \left\{\operatorname{dim}_{K}\left(V^{P}\right)+2, \operatorname{dim}_{K}(V)\right\}$.
\end{abstract}

\section{INTRODUCTION}

Let $G$ be a finite group acting linearly on a finite-dimensional vector space $V$ over a field $K$ of characteristic $p$, and assume that $p$ divides the group order, $|G|$, so that $V$ is a modular representation of $G$. We write $R:=S\left(V^{*}\right)$ for the symmetric algebra of the dual of $V$ and $R^{G}$ for the ring of invariants. For an ideal $I$ in $R^{G}$ and an $R^{G}$-module $M$, grade $(I, M)$ denotes the maximal length of an $M$-regular sequence whose elements lie in $I$. The depth of $M$ is grade $\left(R_{+}^{G}, M\right)$, where $R_{+}^{G}$ is the ideal generated by all homogeneous invariants of positive degree. By the AuslanderBuchsbaum formula, $\operatorname{dim}_{K}(V)-\operatorname{depth}\left(R^{G}\right)$ is the projective dimension of $R^{G}$ as a module over a homogeneous system of parameters. Thus $\operatorname{depth}\left(R^{G}\right) \operatorname{determines}$ a measure of the structural complexity of $R^{G}$. However, despite significant recent progress, computing $\operatorname{depth}\left(R^{G}\right)$ remains, in general, a difficult problem.

The cohomology of the group $G$ with coefficients $R, H^{*}(G, R)$ has proven to be a useful tool in determining $\operatorname{depth}\left(R^{G}\right)$. Let $m$ be the least positive integer such that $H^{m}(G, R) \neq 0$. Then we call $c c_{G}(R):=m$ the cohomological connectivity of the $G$-module $R$. In Section 1 we observe that

$$
\operatorname{depth}\left(R^{G}\right) \geq \min \left\{\operatorname{dim}_{K}\left(V^{P}\right)+c c_{G}(R)+1, \operatorname{dim}_{K}(V)\right\},
$$

where $P$ denotes a Sylow $p$-subgroup of $G$. This gives a stronger lower bound than the one obtained by [4]. One might hope that the inequality is actually an equality. In fact, this was proved to be true if $p^{2}$ does not divide $|G|$ (see [10, Theorem 3.1]) or if $G$ is cyclic (see [4] or [3]). However, the following example shows that equality does not hold in general.

Received by the editors July 17, 2003 and, in revised form, December 17, 2003.

2000 Mathematics Subject Classification. Primary 13A50, 20J06, 13C15.

This research was supported by EPSRC grant GR/R32055/01. 
Example 0.1. Suppose that $q$ is a power of $p$ and that $\mathbf{F}_{q} \subseteq K$. Consider the group $G=U_{n}\left(\mathbf{F}_{q}\right)$ of all $n \times n$ upper triangular matrices with entries in $\mathbf{F}_{q}$ and ones on the diagonal. $G$ is a $p$-group, and for the natural action on $V=K^{n}$ we have $\operatorname{dim}\left(V^{G}\right)=$ 1. Moreover, $H^{1}(G, K) \neq 0$, so $c c_{G}(R)=1$, since $H^{1}(G, K)$ is embedded into $H^{1}(G, R)$ via the identification of $K$ with the homogeneous component of degree 0 of $R$. Thus $\operatorname{dim}_{K}\left(V^{G}\right)+c c_{G}(R)+1=3$. But it is well known that $R^{G}$ is isomorphic to a polynomial ring (see [13] Proposition 5.5.5]). In particular, $R^{G}$ is CohenMacaulay, so $\operatorname{depth}\left(R^{G}\right)=n$. For $n>3$, this is greater than $\operatorname{dim}_{K}\left(V^{G}\right)+c c_{G}(R)+$ 1.

This paper is primarily concerned with identifying representations for which equality holds. Extending the terminology used in [10, we will call $R^{G}$ flat if

$$
\operatorname{depth}\left(R^{G}\right)=\min \left\{\operatorname{dim}_{K}\left(V^{P}\right)+c c_{G}(R)+1, \operatorname{dim}_{K}(V)\right\} .
$$

As a consequence of (1), the ring $R^{G}$ is simultaneously flat and Cohen-Macaulay if and only if $c c_{G}(R)+1 \geq \operatorname{codim}_{K}\left(V^{P}\right)$. Thus, throughout the paper, we will assume $c c_{G}(R)+1<\operatorname{codim}_{K}\left(V^{P}\right)$.

As a matter of convenience we will assume that $K$ is algebraically closed. Since extending $K$ does not change the depth of $R^{G}$, this assumption does not limit the scope of our results.

In Section 1 we prove (1) and introduce an ideal $\mathfrak{i}$ (see also [6] and [7]) which plays a crucial role in the paper. In Section 2 we characterize flatness and give two conditions which together guarantee flatness. In Section 3 and Section 4 we explore these two conditions. In Section 5 we apply our results to groups with cyclic Sylow $p$-subgroup. In particular we prove that if $G$ is $p$-nilpotent and $P$ is cyclic, then

$$
\operatorname{depth}\left(R^{G}\right)=\min \left\{\operatorname{dim}_{K}\left(V^{P}\right)+2, \operatorname{dim}_{K}(V)\right\} .
$$

In Section 6 we give several examples of flat $R^{G}$ for $G$ with non-cyclic Sylow $p$ subgroup. In Section 7 we use a spectral sequence, analogous to that used by Ellingsrud and Skjelbred 4, to prove a technical result required in Section 2

\section{The INEQUALITY AND THE IDEAL}

For a prime ideal $\mathfrak{P} \in \operatorname{Spec}(R)$, define the inertia subgroup as

$$
G_{\mathfrak{P}}:=\{g \in G \mid g(f)-f \in \mathfrak{P} \text { for all } f \in R\} .
$$

Fleischmann and Shank [7, Theorem 2.4(ii)] proved that if $p$ does not divide the index $\left[G: G_{\mathfrak{P}}\right]$, then

$$
\operatorname{depth}\left(R^{G}\right)=\operatorname{dim}(R / \mathfrak{P})+\operatorname{grade}\left(\mathfrak{p}, R^{G}\right),
$$

where $\mathfrak{p}=R^{G} \cap \mathfrak{P}$.

Assume that $p$ does not divide the index $\left[G: G_{\mathfrak{P}}\right]$. Then there is a Sylow $p$ subgroup $P \leq G$, which is contained in $G_{\mathfrak{P}}$. Let $V^{P}$ denote the fixed space of $P$ in $V$ and take $\mathfrak{I}:=\mathcal{I}\left(V^{P}\right) \subset R$ to be the ideal generated by all linear forms in $V^{*}$ that vanish on $V^{P}$. Then $\mathfrak{I}$ is a prime ideal which can be described as follows:

Lemma 1.1. $\mathfrak{I}=\left((g-1) V^{*} \mid g \in P\right) R \subset \mathfrak{P}$.

Proof. Suppose $g \in G$. Clearly $(g-1) V^{*} \subseteq \mathcal{I}\left(V^{g}\right)$. Since the rank of a matrix is equal to the rank of its transpose, $\operatorname{rank}\left(\left.(g-1)\right|_{V^{*}}\right)=\operatorname{codim}\left(V^{g}\right)$. Thus $\mathcal{I}\left(V^{g}\right)=$ $\left((g-1) V^{*}\right) R$. Furthermore, $V^{P}=\bigcap_{g \in P} V^{g}$. Thus, using the fact that the ideal 
associated to an intersection of varieties is the sum of the ideals associated to the varieties, we have $\mathcal{I}\left(V^{P}\right)=\left(\sum_{g \in P}(g-1) V^{*}\right) R=\left((g-1) V^{*} \mid g \in P\right) R$.

On the other hand, obviously $P \subseteq G_{\Im}$, so $p$ does not divide $\left[G: G_{\Im}\right]$. This shows that $\mathfrak{I}$ is minimal among prime ideals of $R$, to which the hypothesis of (21) applies. Define $\mathfrak{i}:=R^{G} \cap \mathfrak{I}$. Note that, while $\mathfrak{I}$ may depend on the choice of Sylow p-subgroup, $\mathfrak{i}$ does not. Clearly

$$
\operatorname{ht}(\mathfrak{I})=\operatorname{dim}_{K}(V)-\operatorname{dim}_{K}\left(V^{P}\right) .
$$

Hence (2) gives

$$
\operatorname{depth}\left(R^{G}\right)=\operatorname{dim}_{K}\left(V^{P}\right)+\operatorname{grade}\left(\mathfrak{i}, R^{G}\right) .
$$

Using [9, Theorem 1.4], for any proper ideal $I \varsubsetneqq R^{G}$ we have

$$
\operatorname{grade}\left(I, R^{G}\right) \geq \min \left\{c c_{G}(R)+1, \operatorname{ht}(I)\right\} .
$$

Applying (3) and (4) with $I=\mathfrak{i}$ yields

$$
\operatorname{depth}\left(R^{G}\right) \geq \operatorname{dim}_{K}\left(V^{P}\right)+\min \left\{c c_{G}(R)+1, \operatorname{ht}(\mathfrak{i})\right\} .
$$

Observing that $\operatorname{ht}(\mathfrak{i})=\operatorname{ht}(\mathfrak{I})=\operatorname{dim}_{K}(V)-\operatorname{dim}_{K}\left(V^{P}\right)$ proves the following.

Theorem 1.2. $\operatorname{depth}\left(R^{G}\right) \geq \min \left\{\operatorname{dim}_{K}\left(V^{P}\right)+c c_{G}(R)+1, \operatorname{dim}_{K}(V)\right\}$.

\section{Characterizing Flatness}

The following theorem, which combines the scope of [7] with the generality of [10, Theorem 1.5], underpins our characterization of flatness.

Theorem 2.1. Let $g_{1}, g_{2}, \ldots, g_{d} \in R^{G}$ be such that the images $\overline{g_{1}}, \overline{g_{2}}, \ldots, \overline{g_{d}}$ form a homogeneous system of parameters in the quotient ring $R^{G} /$ i. Let $\mathcal{M}$ be a module over the ring $\operatorname{End}_{K G}(R)$ such that $\left(g_{1}, g_{2}, \ldots, g_{d}\right) \mathcal{M} \neq \mathcal{M}$. Then $\left(g_{1}, g_{2}, \ldots, g_{d}\right)$ is a regular $\mathcal{M}$-sequence in $R^{G}$. In particular $\operatorname{depth}_{R^{G}}(\mathcal{M}) \geq d=\operatorname{dim}_{K}\left(V^{P}\right)$.

Proof. First we prove the result for the special case $G=P$. Let $W:=\left(V^{G}\right)^{\perp}=$ $\left\{f \in V^{*}|f|_{V^{G}}=0\right\}$. Note that $P$ acts trivially on the quotient $V^{*} / W$. Hence we can apply [10, Proposition 1.2] with $N=G=P$, which shows that for each $i$ there is a map $\Psi_{i} \in \operatorname{End}_{K G}(R)$, commuting with the action of the elements $g_{1}, \ldots, g_{i-1}, g_{i+1}, \ldots, g_{d}$, and satisfying $\Psi_{i}\left(g_{i} r\right)=r$ for every $r \in R$. In other words $\Psi_{i}$ is a left inverse to the multiplication map $\mu_{g_{i}}: R \rightarrow R, r \mapsto g_{i} r$. Suppose $\left(g_{1}, g_{2}, \ldots, g_{s}\right)$ is a regular sequence on $\mathcal{M}$ with $s<d$, and suppose $m \in \mathcal{M}$ with $g_{s+1}(m) \in\left(g_{1}, g_{2}, \ldots, g_{s}\right) \mathcal{M}$. Then $m=\Psi_{s+1}\left(g_{s+1} m\right) \in\left(g_{1}, g_{2}, \ldots, g_{s}\right) \mathcal{M}$, proving that $\left(g_{1}, g_{2}, \ldots, g_{s+1}\right)$ is $\mathcal{M}$-regular as well. Hence $d=\operatorname{dim}\left(R^{G} / \mathfrak{i}\right)=\operatorname{dim}_{K}\left(V^{P}\right) \leq$ $\operatorname{depth}_{R^{P}}(\mathcal{M})$.

Now let $G$ be arbitrary, let $P$ be a Sylow $p$-subgroup and let $\mathfrak{i}_{P}:=\mathfrak{I} \cap R^{P}$ with $\mathfrak{i}=$ $\mathfrak{i}_{P} \cap R^{G}$. Moreover let $\mathcal{E}:=\operatorname{End}_{K}(R)$. Thus $\operatorname{End}_{K H}(R)=\mathcal{E}^{H}$ for every subgroup $H \leq G$. Note that the surjective relative transfer operator $\operatorname{Tr}_{P}^{G}: \mathcal{E}^{P} \rightarrow \mathcal{E}^{G}$ induces a splitting of $\mathcal{E}^{G}$-modules $\mathcal{E}^{P}=\mathcal{E}^{G} \oplus \mathcal{C}$. Hence $\mathcal{N}:=\mathcal{E}^{P} \otimes_{\mathcal{E}^{G}} \mathcal{M}$ is an $\mathcal{E}^{P}$-module, such that the restricted $\mathcal{E}^{G}$-module has $\mathcal{M}$ as a direct summand. Assume that $g_{1}, g_{2}, \ldots, g_{d}$ are elements such that the images $\overline{g_{1}}, \overline{g_{2}}, \ldots, \overline{g_{d}}$ form a homogeneous system of parameters in the quotient ring $R^{G} / \mathfrak{i}$. Since $R^{P} / \mathfrak{i}_{P}$ is a finite extension of $R^{G} / \mathfrak{i}$, these images also form a homogeneous system of parameters in $R^{P} / \mathfrak{i}_{P}$, and therefore the originals form an $\mathcal{N}$-regular sequence. Since $\mathcal{M}$ is a direct summand of $\mathcal{N}_{\mid \mathcal{E}^{G}}$, the sequence $\left(g_{1}, g_{2}, \ldots, g_{d}\right)$ is also $\mathcal{M}$-regular. 
Corollary 2.2. For every non-zero element $\alpha \in H^{m}(G, R)$ one has $\operatorname{Ann}_{R^{G}}(\alpha) \leq \mathfrak{i}$.

Proof. It is obvious from the definition of cohomology that $H^{m}(G, R)$ is a module over $\operatorname{End}_{K G}(R)$. Let $x \in R^{G} \backslash \mathfrak{i}$. Then $\bar{x}$ is contained in a homogeneous system of parameters of $R^{G} / \mathfrak{i}$ and therefore acts regularly on $H^{m}(G, R)$. Hence $x \notin \operatorname{Ann}_{R^{G}}(\alpha)$.

The following conceptual characterization of flatness in terms of the prime ideal $\mathfrak{i}$ will guide further investigation.

Theorem 2.3. Assume that $m:=c c_{G}(R)<\operatorname{codim}_{K}\left(V^{P}\right)-1$. Then the following are equivalent:

(a) $R^{G}$ is flat;

(b) the ideal $\mathfrak{i}$ is an associated prime of the $R^{G}$-module $H^{m}(G, R)$;

(c) $H^{m}(G, R)$ contains an $R^{G}$-submodule of Krull dimension $\operatorname{dim}_{K}\left(V^{P}\right)$.

If these conditions are satisfied, then $\operatorname{depth}_{R^{G}}\left(H^{m}(G, R)\right)=\operatorname{dim}_{K}\left(V^{P}\right)$.

Proof. (a) $\Rightarrow$ (b): From Theorem 7.1 we see that grade $\left(\mathfrak{i}, R^{G}\right)=m+1$ implies that grade $\left(\mathfrak{i}, H^{m}(G, R)\right)=0$, hence $\mathfrak{i}$ consists of zero-divisors on $H^{m}(G, R)$ and therefore lies in some associated prime $\mathrm{p}:=\operatorname{Ann}_{R^{G}}(\alpha)$ with $\alpha \in H^{m}(G, R) \backslash\{0\}$. On the other hand, Corollary 2.2 implies that $\operatorname{Ann}_{R^{G}}(\alpha) \subseteq \mathfrak{i}$. Hence $\mathfrak{i}=\mathrm{p}$.

(b) $\Rightarrow$ (c): Take the submodule generated by $\alpha$.

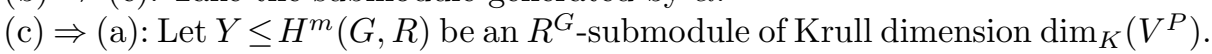
Then there is an associated prime p of $Y$ such that the Krull dimensions of the modules $R^{G} / \mathrm{p}, Y$ and $R^{G} / \mathfrak{i}$ coincide. Again Corollary 2.2 shows that $\mathrm{p}=\mathfrak{i}$, which implies that grade $\left(\mathfrak{i}, H^{m}(G, R)\right)=0$. Hence grade $\left(\mathfrak{i}, R^{G}\right)=m+1$, by Theorem 7.1 If $\mathfrak{i}$ is an associated prime of $H^{m}(G, R)$, then the $R^{G}$-depth of this module is less than or equal to $\operatorname{dim} R^{G} / \mathfrak{i}=\operatorname{dim}_{K}\left(V^{P}\right)$ (see 2, Proposition 1.2.13]). On the other hand it follows from [10, Theorem 2.13]), or from Corollary 2.2 that this depth is also greater than or equal to $\operatorname{dim}_{K}\left(V^{P}\right)$.

While we are aware of a number of instances where the existence of a suitable positive degree cohomology class provides a sufficient condition determining the depth of $R^{G}$, we believe that Theorem 2.3 is the first example of a theorem giving a condition on the positive degree cohomology which is necessary as well as sufficient.

Suppose $m:=c c_{G}(R)$ and $\alpha \in H^{m}(G, R) \backslash\{0\}$. Then the variety in $V$ associated to $\operatorname{Ann}_{R^{G}}(\alpha)$ is

$$
\mathcal{V}(\alpha):=\left\{x \in V \mid f(x)=0 \text { for all } f \in \operatorname{Ann}_{R^{G}}(\alpha)\right\} .
$$

Similarly the variety in $V$ associated to the prime ideal $\mathfrak{i}=\mathfrak{I} \cap R^{G}$ is

$$
\mathcal{V}(\mathfrak{i})=\bigcup_{P^{\prime}} V^{P^{\prime}}
$$

where the union runs through all Sylow $p$-subgroups $P^{\prime} \leq G$. The condition $\mathcal{V}(\alpha) \subseteq \bigcup_{P^{\prime}} V^{P^{\prime}}$ implies $\mathfrak{i} \subseteq \sqrt{\operatorname{Ann}_{R^{G}}(\alpha)}$ and therefore, by Corollary 2.2. we have equality in both inclusions. Using this we obtain the following geometric version of Theorem 2.3.

Theorem 2.4. Assume that $m:=c c_{G}(R)<\operatorname{codim}_{K}\left(V^{P}\right)-1$ and $\alpha \in H^{m}(G, R) \backslash$ $\{0\}$. Then the following are equivalent:

(a) $\mathcal{V}(\alpha) \subseteq \mathcal{V}(\mathfrak{i})$;

(b) $\mathcal{V}(\alpha)=\mathcal{V}(\mathfrak{i})$; 
(c) $\sqrt{\operatorname{Ann}_{R^{G}}(\alpha)}=\mathfrak{i}$.

Furthermore, $R^{G}$ is flat if and only if there exists $\alpha \in H^{m}(G, R) \backslash\{0\}$ satisfying (a), (b) or (c).

Proof. Using Theorem 2.3 it suffices to note that $\sqrt{\operatorname{Ann}_{R^{G}}(\alpha)}=\mathfrak{i}$ implies that $\mathfrak{i}$ is an associated prime of $H^{m}(G, R)$. However this is a standard fact about Noetherian rings and modules.

In order to apply Theorem 2.4 we need to find classes $\alpha \in H^{m}(G, R) \backslash\{0\}$ satisfying $\mathcal{V}(\alpha) \subseteq \mathcal{V}(\mathfrak{i})$ or, equivalently, $\mathfrak{i} \subseteq \sqrt{\operatorname{Ann}_{R^{G}}(\alpha)}$. By [12, Lemma 1.3], elements in the image of the relative transfer, $\operatorname{Tr}_{H}^{G}$, annihilate cohomology classes in the kernel of the restriction, $\operatorname{res}_{G, H}$, and, by [6, 12.5], $\mathfrak{i}$ is the radical of a sum of relative transfer ideals.

Recall that for a subgroup $H \leq G$ the relative transfer is the $R^{G}$-module homomorphism $\operatorname{Tr}_{H}^{G}: R^{H} \rightarrow R^{G}, r \mapsto \sum_{g} g(r)$, where $g$ runs through a set of representatives of $H$-cosets satisfying $G=\uplus g H$. This definition is independent of the choice of representatives and the image $\operatorname{Tr}_{H}^{G}\left(R^{H}\right)$ is an ideal in $R^{G}$. For any set $\chi$ of subgroups of $G$ we define

$$
\mathfrak{i}_{\chi}:=\sum_{X \in \chi} \operatorname{Tr}_{X}^{G}\left(R^{X}\right) \triangleleft R^{G} .
$$

This is a proper ideal of $R^{G}$ if and only if $p$ divides all indices $[G: X]$ for $X \in \chi$. The varieties of relative transfer ideals $\operatorname{Tr}_{H}^{G}\left(R^{H}\right)$ have been described in 6 , Theorem 12.4] and [12, Lemma 1.1]:

$$
\mathcal{V}\left(\operatorname{Tr}_{H}^{G}\left(R^{H}\right)\right)=\left\{v \in V|p|\left[G_{v}: G_{v} \cap{ }^{g} H\right] \text { for all } g \in G\right\},
$$

where ${ }^{g} H:=g H g^{-1}$. Note that, if $p$ divides $[G: H]$, then $H$ does not contain a Sylow $p$-subgroup of $G$ and $\mathcal{V}(i) \subseteq \mathcal{V}\left(\operatorname{Tr}_{H}^{G}\left(R^{H}\right)\right)$. Therefore $\sqrt{\mathfrak{i}_{\chi}} \subseteq \mathfrak{i}$ whenever $\sqrt{\mathfrak{i}_{\chi}} \varsubsetneqq R^{G}$. Furthermore, $\mathfrak{i}=\sqrt{\mathfrak{i}_{p-\text { prop }}}$, where "p-prop" denotes the set of all proper subgroups of Sylow p-subgroups of $G$ (see [6, 12.5] or Theorem 3.1 below). For a family $\chi$ of subgroups of $G$, define

$$
\mathcal{K}_{\chi}^{j}:=\mathcal{K}_{\chi}^{j}(G):=\bigcap_{X \in \chi} \operatorname{ker}\left(\left.\left(\operatorname{res}_{G, X}\right)\right|_{H^{j}(G, R)}\right)
$$

and $\mathcal{K}_{\chi}:=\bigoplus_{j>1} \mathcal{K}_{\chi}^{j}$. The following lemma is an immediate consequence of [12, Lemma 1.3].

Lemma 2.5. For each family $\chi$ of subgroups of $G$ one has

$$
\mathfrak{i}_{\chi} \subseteq \operatorname{Ann}_{R^{G}}\left(\mathcal{K}_{\chi}\right) .
$$

This leads to the following criterion of flatness.

Theorem 2.6. For $m=c c_{G}(R)$ and $\chi$ a family of subgroups of $G$, if $\sqrt{\mathfrak{i}_{\chi}}=\mathfrak{i}$ and $\mathcal{K}_{\chi}^{m} \neq 0$, then $R^{G}$ is flat.

Proof. Lemma 2.5 and $\mathcal{K}_{\chi}^{m} \neq 0$ imply $\sqrt{\mathfrak{i}_{\chi}} \subseteq \sqrt{\operatorname{Ann}_{R^{G}}(\alpha)} \subseteq \mathfrak{i}$. Using the first condition gives $\sqrt{\operatorname{Ann}_{R^{G}}(\alpha)}=\mathfrak{i}$. Hence the hypothesis (c) of Theorem 2.4 holds. 


\section{The GEOMETRY OF THE IDEAL $\mathfrak{i}$}

In this section we use the geometry of $\mathfrak{i}$ to investigate families of subgroups, $\chi$, for which $\sqrt{i_{\chi}}=\mathfrak{i}$. This is an important first step in the use of Theorem 2.6 Since $\operatorname{Tr}_{{ }^{g} H}^{G}\left(R^{g} H\right)=\operatorname{Tr}_{H}^{G}\left(R^{H}\right)$ for every $g \in G$ and $\operatorname{Tr}_{H}^{G}\left(\operatorname{Tr}_{U}^{H}\left(R^{U}\right)\right)=\operatorname{Tr}_{U}^{G}\left(R^{U}\right)$ for $U \leq H$, the ideal $\sqrt{i_{\chi}}$ does not change if we adjoin to $\chi$ all subgroups of conjugates of $X \in \chi$. Similarly $\mathcal{K}_{\chi}$ does not change either, hence we can, if we wish, assume that $\chi$ is closed under taking conjugates and subgroups. Let $\chi_{p}^{\prime}$ denote the set of $p$-subgroups of $G$ not in $\chi$.

Theorem 3.1. If $\chi$ is closed under taking conjugates and subgroups, then

$$
\mathcal{V}\left(\mathfrak{i}_{\chi}\right)=\left\{v \in V|p|\left[G_{v}: G_{v} \cap X\right] \text { for all } X \in \chi\right\}=\bigcup_{Q \in \chi_{p}^{\prime}} V^{Q} .
$$

Proof. The first equality follows from (6) using

$$
\mathcal{V}\left(\mathfrak{i}_{\chi}\right)=\mathcal{V}\left(\sum_{X \in \chi} \operatorname{Tr}_{X}^{G}\left(R^{X}\right)\right)=\bigcap_{X \in \chi} \mathcal{V}\left(\operatorname{Tr}_{X}^{G}\left(R^{X}\right)\right) .
$$

We now prove the second equality. To show the first inclusion, suppose $v \in \mathcal{V}\left(\mathfrak{i}_{\chi}\right)$ and let $P_{v}$ denote a Sylow $p$-subgroup of the isotropy subgroup $G_{v}$. Since $p$ divides $\left[G_{v}: G_{v} \cap X\right]$ for all $X \in \chi$, it is clear that $P_{v} \notin \chi$. Thus $v \in V^{P_{v}} \subseteq \bigcup_{Q \in \chi_{p}^{\prime}} V^{Q}$.

To show the reverse inclusion, let $v \in V^{Q}$ for some $Q \in \chi_{p}^{\prime}$ and assume, by way of contradiction, that $v \notin \mathcal{V}\left(\mathfrak{i}_{\chi}\right)$. Then there exists $H \in \chi$ such that $p$ does not divide $\left[G_{v}: G_{v} \cap H\right]$. Thus $H$ contains a Sylow $p$-subgroup of $G_{v}$, say $P_{v}$. Since $\chi$ is closed under taking subgroups, $P_{v} \in \chi$. However, $Q$ is a $p$-subgroup of $G_{v}$. Therefore some conjugate of $Q$ is contained in $P_{v}$. Again, since $\chi$ is closed under conjugation and taking subgroups, we arrive at the contradiction $Q \in \chi$.

Corollary 3.2. For $j \in\{1,2\}$, let $\chi_{j}$ be closed under conjugation and taking subgroups. Then

$$
\mathcal{V}\left(\mathfrak{i}_{\chi_{1}}\right) \subseteq \mathcal{V}\left(\mathfrak{i}_{\chi_{2}}\right)
$$

if and only if the following holds:

For each p-group $Q \in \chi_{2} \backslash \chi_{1}$ there exists $S \in\left(\chi_{2}\right)_{p}^{\prime}$, such that $Q \varsubsetneqq S$ and

$$
V^{Q}=V^{S} \text {. }
$$

Proof. " $\Rightarrow$ ": Suppose $\mathcal{V}\left(\mathfrak{i}_{\chi_{1}}\right) \subseteq \mathcal{V}\left(\mathfrak{i}_{\chi_{2}}\right)$ and $Q$ is a $p$-group in $\chi_{2} \backslash \chi_{1}$. Applying Theorem 3.1 gives $V^{Q} \subseteq \mathcal{V}\left(\mathfrak{i}_{\chi_{1}}\right)$. Thus $V^{Q} \subseteq \mathcal{V}\left(\mathfrak{i}_{\chi_{2}}\right)$. Hence for each $v \in V^{Q}, p$ divides $\left[G_{v}: G_{v} \cap Q\right]$ and there is a Sylow $p$-subgroup of $G_{v}$, say $P_{v}$, with $Q \varsubsetneqq P_{v}$. As $v \in \mathcal{V}\left(\mathfrak{i}_{\chi_{2}}\right)$, using the first equality of Theorem 3.1 we have $P_{v} \notin \chi_{2}$. Clearly for any $v \in V$ we have $V^{P_{v}} \subset V^{Q}$. Furthermore $V^{Q} \subseteq \bigcup_{v \in V^{Q}} V^{P_{v}}$. Thus, since the finite group $G$ has only a finite number of subgroups, $V^{Q}=\bigcup_{v \in \mathcal{S}} V^{P_{v}}$ for some finite $\mathcal{S} \subseteq V^{Q}$. Since $K$ is algebraically closed and therefore an infinite field, we conclude $V^{Q}=V^{P_{v^{\prime}}}$ for some $Q \varsubsetneqq P_{v^{\prime}} \in\left(\chi_{2}\right)_{p}^{\prime}$.

" $\Leftarrow$ ": From Theorem 3.1

$$
\mathcal{V}\left(\mathfrak{i}_{\chi_{1}}\right)=\bigcup_{Q \in\left(\chi_{1}\right)_{p}^{\prime}} V^{Q}
$$


However, $\left(\chi_{1}\right)_{p}^{\prime}$ is the disjoint union of $\left(\chi_{1}\right)_{p}^{\prime} \cap\left(\chi_{2}\right)_{p}^{\prime}$ and $\left(\chi_{1}\right)_{p}^{\prime} \cap \chi_{2}$. If $Q \in\left(\chi_{1}\right)_{p}^{\prime} \cap \chi_{2}$, then by hypothesis $V^{Q}=V^{S}$ for some $S \in\left(\chi_{2}\right)_{p}^{\prime}$. Therefore, for any $Q \in\left(\chi_{1}\right)_{p}^{\prime}$, $V^{Q} \subseteq \bigcup_{S \in\left(\chi_{2}\right)_{p}^{\prime}} V^{S}$ and the result follows from Theorem 3.1

Lemma 3.3. Let $P$ be a Sylow p-subgroup of $G$ and let $Q$ be a proper subgroup of $P$. Define $G_{V^{Q}}:=\left\{g \in G \mid g w=w \forall w \in V^{Q}\right\}$. Then $V^{P} \varsubsetneqq V^{Q}$ if and only if $p \mid\left[G: G_{V^{Q}}\right]$.

Proof. First observe that $V^{P} \subseteq V^{Q}$ and $Q \leq G_{V^{Q}}$.

Suppose $p$ does not divide $\left[G: G_{V^{Q}}\right]$. Then $G_{V^{Q}}$ contains a Sylow $p$-subgroup of $G$, say $P^{\prime}$. Thus $V^{P} \subseteq V^{Q} \subseteq V^{P^{\prime}}$. However, $P$ and $P^{\prime}$ are conjugate so $\operatorname{dim}\left(V^{P}\right)=\operatorname{dim}\left(V^{P^{\prime}}\right)$. Therefore $\bar{V}^{P}=V^{Q}$.

Suppose $p \mid\left[G: G_{V^{Q}}\right]$. Then $P \backslash G_{V^{Q}}$ is non-empty and there exists $g \in P$ and $v \in V^{Q}$ with $g v \neq v$. Therefore $V^{P} \varsubsetneqq V^{Q}$.

In the following we adopt the notation $X \leq_{G} H\left(X<_{G} H\right)$ to mean ${ }^{g} X \leq H \leq$ $G\left({ }^{g} X<H\right)$ for some $g \in G$.

Proposition 3.4. Let $H_{1}, H_{2}, \ldots, H_{k} \leq G$ with $p \mid\left[G: H_{i}\right]$ for all $i$ and let $P$ be a fixed Sylow p-subgroup of $G$. Then the following are equivalent:

(a) $\mathfrak{i}=\sqrt{\mathfrak{i}_{\left\{H_{1}, H_{2}, \ldots, H_{k}\right\}}}$;

(b) $\forall Q<P$ with $V^{P} \varsubsetneqq V^{Q}: Q \leq_{G} H_{i}$ for some $i \in\{1, \ldots, k\}$;

(c) $\forall Q<P$ with $p \mid\left[G: G_{V^{Q}}\right]: Q \leq_{G} H_{i}$ for some $i \in\{1, \ldots, k\}$.

Proof. Let $\chi_{1}:=\left\{X \leq G \mid \exists i \in\{1, \ldots, k\}: X \leq_{G} H_{i}\right\}$ and $\chi_{2}:=\left\{Q \leq G \mid Q<_{G}\right.$ $P\}$. From Theorem 3.1 and (5) we see that $\mathcal{V}\left(\mathfrak{i}_{\chi_{2}}\right)=\mathcal{V}(\mathfrak{i})$. From Theorem 3.1 and (6) we see that $\left.\mathcal{V}\left(\mathfrak{i}_{\chi_{1}}\right)=\mathcal{V}\left(\mathfrak{i}_{\left\{H_{1}\right.}, H_{2}, \ldots, H_{k}\right\}\right)$. Moreover, since all $H_{i}$ 's have index divisible by $p$, we know that $\mathcal{V}(\mathfrak{i}) \subseteq \mathcal{V}\left(\mathfrak{i}_{\chi_{1}}\right)$. From Corollary 3.2 and Lemma 3.3 we see that the reverse inclusion is equivalent to either one of (b) or (c). Now the statement follows from Hilbert's Nullstellensatz.

\section{Remark 3.5.}

(a) For any subgroup $H \leq G$ we have $\mathfrak{i}=\sqrt{\mathfrak{i}_{H}}$ if and only if every $p$-subgroup $Q \leq G$ with $p \mid\left[G: G_{V^{Q}}\right]$ satisfies $Q \leq_{G} H$. If $H$ is a normal subgroup, then this condition is equivalent to the fact that $H$ contains all $p$-elements $g \in G$ such that $p$ divides the index $\left[G: G_{V^{g}}\right]$, where $G_{V^{g}}$ is the pointwise stabilizer of the fixed point space of $g$. In particular the subgroup $M:=$ $\langle g \in G| g$ a $p$ - element with $p\left|\left[G: G_{V^{g}}\right]\right\rangle \triangleleft G$ is the unique minimal normal subgroup of $G$ with $\mathfrak{i}=\sqrt{\mathfrak{i}_{M}}$.

(b) Let $P$ be a fixed Sylow $p$-subgroup of $G$. Without loss of generality, we can assume that the subgroups $H_{1}, H_{2}, \ldots, H_{k}$ appearing in Proposition 3.4 are proper subgroups of $P$. Indeed, by Mackey's formula we have for each $r \in R^{H_{i}}: \operatorname{Tr}_{H_{i}}^{G}(r)=\sum_{g} \operatorname{Tr}_{P \cap}^{P} g_{H_{i}}(g r)$, where $g$ runs through a system of representatives of double cosets $P g H_{i}$ in $G$. Hence

$$
[G: P] \operatorname{Tr}_{H_{i}}^{G}(r)=\operatorname{Tr}_{P}^{G}\left(\operatorname{Tr}_{H_{i}}^{G}(r)\right)=\sum_{g} \operatorname{Tr}_{P \cap{ }^{g} H_{i}}^{G}(g r)
$$

and we see that

$$
\sqrt{\mathfrak{i}_{\left\{H_{1}, H_{2}, \ldots, H_{k}\right\}}}=\sqrt{\mathfrak{i}_{\left\{P \cap g_{H_{i}} \mid g \in G\right\}}} .
$$


Example 3.6. Suppose that we are in the "mildly modular" case, i.e., $p^{2}$ does not divide $|G|$. Take a non-identity $p$-element $g \in G$. Then $\langle g\rangle \leq G$ has order $p$ and therefore is a Sylow $p$-subgroup. But we certainly have $\langle g\rangle \leq G_{V^{g}}$, hence the index $\left[G: G_{V^{g}}\right]$ is not divisible by $p$. Therefore, by Proposition 3.4 we can take $\chi=\{1\}$ in Theorem 2.6. Since the restriction is non-injective, $\mathcal{K}_{\chi}^{m} \neq 0$. Thus $R^{G}$ is flat and we have recovered Theorem 3.1 of [10].

\section{Restricting COHOMOlOGy}

Our primary tool for identifying flat representations is Theorem 2.6. Proposition 3.4 allows us to identify suitable families of subgroups. In this section we address the problem of identifying a suitable non-zero cohomology class. It is convenient to assume that the elements of $\chi=\left\{H_{1}, \ldots, H_{k}\right\}$ are proper subgroups of one Sylow $p$-subgroup, $P$, of $G$. We can then attempt to determine the existence of $\alpha \in \mathcal{K}_{\chi}^{m} \backslash\{0\}$ locally, i.e. on the level of $P$. In principle this can be done, using the fact that the restriction map $\operatorname{res}_{G, P}: H^{*}(G, R) \rightarrow H^{*}(P, R)$ is injective and its image consists of the stable cohomology

$H^{*}(P, R)_{G-s t a b}:=\left\{\eta \in H^{*}(P, R) \mid \operatorname{res}_{P, P \cap{ }^{P} P}(\eta)=\operatorname{res}_{g_{P}, P \cap{ }^{g} P}(g \eta), g \in P \backslash G / P\right\}$,

where $P \backslash G / P$ denotes a system of $P-P$ double coset representatives (see 1 . Proposition 3.8.2] or [5] Corollary 4.2.7]). Note also, that the transfer (or corestriction) $\operatorname{Tr}_{P}^{G}$ from $H^{m}(P, R)$ to $H^{m}(G, R)$ induces an isomorphism $H^{m}(P, R)_{G-s t a b} \cong$ $H^{m}(G, R)$.

Lemma 4.1. Let $P$ be a Sylow p-subgroup of $G$ with normalizer quotient $N:=$ $N_{G}(P) / P$, let $\chi:=\left\{Q_{1}, Q_{2}, \ldots, Q_{k}\right\}$ be a family of proper subgroups of $P$ and let

$$
\hat{\chi}:=\chi \cup\left\{P \cap^{g} P \mid g \in G \backslash N_{G}(P)\right\} .
$$

Then

$$
\begin{aligned}
H^{m}(P, R)^{N} \cap \mathcal{K}_{\hat{\chi}}(P) & =H^{m}(P, R)_{G-s t a b} \cap \mathcal{K}_{\hat{\chi}}(P) \\
& \subseteq H^{m}(P, R)_{G-s t a b} \cap \mathcal{K}_{\chi}(P) \cong \mathcal{K}_{\chi}^{m}(G),
\end{aligned}
$$

where the last isomorphism is induced by transfer and restriction.

Proof. Since $H^{m}(P, R)_{G-s t a b} \cong H^{m}(G, R)$ and $G$ acts trivially on $H^{m}(G, R)$, we have $H^{m}(P, R)_{G-s t a b} \subseteq H^{m}(P, R)^{N}$ and $H^{m}(P, R)_{G-s t a b} \cap \mathcal{K}_{\hat{\chi}}(P) \subseteq H^{m}(P, R)^{N} \cap$ $\mathcal{K}_{\hat{\chi}}(P)$. Suppose $\eta \in H^{m}(P, R)^{N} \cap \mathcal{K}_{\hat{\chi}}(P)$ and $g \in G$. If $g \in N_{G}(P)$, then the stability condition becomes $\eta=g \eta$ and is satisfied because $\eta \in H^{m}(P, R)^{N}$. If $g \in$

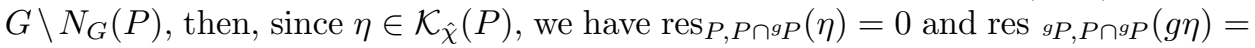
$g\left(\operatorname{res}_{P, g^{-1} P g \cap P}(\eta)\right)=0$. Thus again the stability condition is satisfied. Therefore $\eta \in H^{m}(P, R)_{G-s t a b}$ and $H^{m}(P, R)^{N} \cap \mathcal{K}_{\hat{\chi}}(P)=H^{m}(P, R)_{G-s t a b} \cap \mathcal{K}_{\hat{\chi}}(P)$.

Since $\chi \subset \hat{\chi}$, we have $\mathcal{K}_{\hat{\chi}}(P) \subseteq \mathcal{K}_{\chi}(P)$. Therefore

$$
H^{m}(P, R)_{G-s t a b} \cap \mathcal{K}_{\hat{\chi}}(P) \subseteq H^{m}(P, R)_{G-s t a b} \cap \mathcal{K}_{\chi}(P) .
$$

Suppose $\rho \in H^{m}(P, R)_{G-s t a b} \cap \mathcal{K}_{\chi}(P)$. Using the Mackey formula in cohomology (see [1, Proposition 3.6.16]), we get

$$
\operatorname{res}_{G, Q_{i}}\left(\operatorname{Tr}_{P}^{G}(\rho)\right)=\sum_{g \in Q_{i} \backslash G / P} \operatorname{Tr}_{Q_{i} \cap{ }^{Q_{P}}}^{Q_{i}}\left(\operatorname{res} g_{P, Q_{i} \cap g_{P}}(g \rho)\right)=0,
$$


because

$$
\begin{aligned}
\operatorname{res}{ }^{g_{P}}, Q_{i} \cap{ }^{g} P(g \rho) & =\operatorname{res}_{P \cap}{ }^{{ }} P, Q_{i} \cap{ }^{{ }_{P}}\left(\operatorname{res}{ }^{g_{P}}, P \cap{ }^{{ }} P(g \rho)\right) \\
& =\operatorname{res}_{P \cap}{ }^{{ }} P, Q_{i} \cap{ }^{{ }_{P}}\left(\operatorname{res}_{P, P \cap}{ }^{g_{P}}(\rho)\right) \\
& =\operatorname{res}_{P, Q_{i} \cap{ }^{g} P}(\rho)=0 .
\end{aligned}
$$

The fact that $\operatorname{res}_{G, P}$ maps $\mathcal{K}_{\chi}^{m}(G)$ onto $H^{m}(P, R)_{G-s t a b} \cap \mathcal{K}_{\chi}(P)$ follows from the transitivity of restriction.

We now describe a way to construct suitable $N$-stable elements in $H^{m}(P, R)^{N} \cap$ $\mathcal{K}_{\hat{\chi}}(P)$, whenever the restricted $P$-module $R_{\mid P}$ contains a suitable direct summand of 'induced type'.

Lemma 4.2. Keep the notation from Lemma 4.1 and let $W$ be a KP-module. Suppose that $\tau \in H^{m}(P, W) \backslash\{0\}$ with $\operatorname{res}_{P, X}(\tau)=0$ for all proper subgroups $X<$ $P$. Assume that the restricted $K P$-module $R_{\mid P}$ has a direct summand, isomorphic to the restricted induced module $\left(W^{\uparrow N_{G}(P)}\right)_{\mid P}$. Then there exists a stable element $\rho \in H^{m}(P, R)^{N} \cap \mathcal{K}_{\hat{\chi}}(P) \backslash\{0\}$.

Proof. Let $i$ and $j$ denote the embeddings of the direct summands

$$
H^{m}(P, W) \hookrightarrow H^{m}\left(P, W^{\uparrow N_{G}(P)} \mid P\right) \text { and } H^{m}\left(P, W^{\uparrow N_{G}(P)} \mid P\right) \hookrightarrow H^{m}(P, R),
$$

respectively, and define $\rho:=j \circ \operatorname{res}_{N_{G}(P), P} \circ \operatorname{Tr}_{P}^{N_{G}(P)} \circ i(\tau)$. Note that the map

$$
\operatorname{Tr}_{P}^{N_{G}(P)} \circ i: H^{m}(P, W) \rightarrow H^{m}\left(N_{G}(P), W^{\uparrow N_{G}(P)}\right)
$$

is an isomorphism, due to the Eckmann-Shapiro lemma. Since $N_{G}(P) / P$ has order prime to $p$, the restriction

$$
\operatorname{res}_{N_{G}(P), P}: H^{m}\left(N_{G}(P), W^{\uparrow N_{G}(P)}\right) \rightarrow H^{m}\left(P, W^{\uparrow N_{G}(P)}\right)^{N}
$$

is an isomorphism (see [5, Exercise 7.2.3. pg 77]) and $j$ is injective. This shows that $\rho$ lies in $H^{m}(P, R)^{N}$ and is non-zero. Moreover

$$
\begin{aligned}
\operatorname{res}_{P, X}(\rho) & =\operatorname{res}_{N_{G}(P), X} \operatorname{Tr}_{P}^{N_{G}(P)}(i(\tau))=\sum_{n \in N} \operatorname{res}_{P, X}(n \tau) \\
& =\sum_{n \in N}{ }^{n}\left(\operatorname{res}_{P,{ }^{-1} X}(\tau)\right)=0
\end{aligned}
$$

Thus $\rho \in H^{m}(P, R)^{N} \cap \mathcal{K}_{\hat{\chi}}(P) \backslash\{0\}$.

Proposition 4.3. Let $P$ be a Sylow p-subgroup of $G$, let $m=c c_{G}(R)$, and let $W$ be a KP-module. Suppose that $\tau \in H^{m}(P, W) \backslash\{0\}$ with $\operatorname{res}_{P, X}(\tau)=0$ for all proper subgroups $X<P$. Suppose that the restricted $K P$-module $R_{\mid P}$ has a direct summand isomorphic to the restricted induced module $\left(W^{\uparrow N_{G}(P)}\right)_{\mid P}$. Then $R^{G}$ is flat.

Proof. Take $\chi$ to be the set of all proper subgroups of $P$. Then by Proposition 3.4 the first condition in Theorem [2.6] is satisfied. The second condition follows from Lemma 4.2 and Lemma 4.1 . 


\section{Cyclic Sylow $p$-Subgroups}

In this section we apply our techniques to groups with cyclic Sylow $p$-subgroup. We start by recording two known facts about group cohomology.

Theorem 5.1. Let $P=\langle\sigma\rangle$ be a cyclic group of order $z$, let $\alpha \in \operatorname{Aut}(P)$ be an automorphism and let $M$ be $a \rtimes \rtimes\langle\alpha\rangle$-module. Then

$$
H^{i}(P, M)= \begin{cases}M^{P} / \operatorname{Tr}^{P}(M) & \text { if } i>0 \text { is even, } \\ \operatorname{ker}\left(\left.\operatorname{Tr}^{P}\right|_{M}\right) /(\sigma-1) M & \text { if } i \text { is odd, }\end{cases}
$$

and the induced (right) action of $\alpha$ on $H^{*}(P, M)$ is given by $\rho^{\alpha}=\alpha^{-1}\left(k^{i} \cdot \rho\right)$, where $\alpha(\sigma)=\sigma^{k}$ with $\operatorname{gcd}(k, z)=1$ and $\operatorname{deg}(\rho) \in\{2 i, 2 i-1\}$.

Proof. See [5, p. 6].

Theorem 5.2. Let $G$ be any group and let $K$ be a field of characteristic $p$. Then

$$
H^{1}(G, K) \cong \operatorname{Hom}(G, \mathbf{Z} / p) \otimes_{\mathbf{F}_{p}} K,
$$

where $\mathbf{F}_{p}$ denotes the prime field of characteristic $p$. In particular $H^{1}(G, K) \neq 0$ if and only if $G$ has a normal subgroup of index $p$.

Proof. See [11, Corollary 10.13].

Throughout the rest of this section we assume that $P$ is a cyclic Sylow $p$-subgroup of $G$. We will use $N$ to denote the normalizer quotient $N_{G}(P) / P$. Recall that a finite group $G$ is called $p$-nilpotent if $G$ has a normal subgroup $H$ of order not divisible by $p$ such that $G / H$ is a $p$-group.

Theorem 5.3. Let $G$ be a finite group with cyclic Sylow p-subgroup P. Then the following are equivalent:

(a) $H^{1}(G, K) \neq 0$;

(b) $G$ is p-nilpotent;

(c) $N_{G}(P)=C_{G}(P)$.

If $G$ acts linearly on a finite-dimensional vector space $V$ over a field $K$ of characteristic $p$ and one of (a), (b) or (c) holds, then $R^{G}$ is flat and

$$
\operatorname{depth}\left(R^{G}\right)=\min \left\{\operatorname{dim}_{K}\left(V^{P}\right)+2, \operatorname{dim}_{K}(V)\right\} .
$$

Proof. By Theorem [5.2, (a) implies that there is $M \triangleleft G$ with $G / M$ of order $p$. Therefore $M \cap P=\varphi(P)$, the Frattini group of $P$ and $M$ is $p$-nilpotent by [8, Chap. IV, 4.7]. Hence $M=(M \cap P) \cdot H$ with normal $p^{\prime}$-subgroup $H$, which in fact is characteristic in $M$ and hence normal in $G$. It follows that $G=P \cdot H$ is $p$-nilpotent. On the other hand, if $G=P \cdot H$ is $p$-nilpotent, then $G / H \cong P$ has a factor group of order $p$, hence $H^{1}(G, K) \neq 0$, by Theorem 5.2 So (a) and (b) are equivalent.

"(b) $\Rightarrow$ (c)": Let $G=P \cdot H$ with normal $p^{\prime}$-complement $H$. Then $N_{G}(P)=$ $P N_{H}(P)=P \times N_{H}(P)=P \times C_{H}(P)=C_{G}(P)$.

"(c) $\Rightarrow$ (a)": This implication is Burnside's theorem (see [8, Chap. IV, 2.6]), which is valid for abelian $P$.

Suppose that $G$ is $p$-nilpotent. We apply Lemma 4.1 with $\hat{\chi}=\chi=\{\varphi(P)\}$ and $m=1$. Notice that $0 \neq H^{1}(G, K) \hookrightarrow H^{1}(G, R)$, where the embedding is induced from the embedding $K \hookrightarrow R=K \oplus R_{+}$. 
From the Lyndon-Hochschild-Serre spectral sequence we get the exact sequence

$$
0 \rightarrow K \cong H^{1}(P / \varphi(P), K) \rightarrow H^{1}(P, K) \rightarrow H^{1}(\varphi(P), K)^{P / \varphi(P)},
$$

where the first map is inflation and the second one is restriction. Let $\eta$ denote a non-zero element of $H^{1}(P / \varphi(P), K)$. Since $N_{G}(P)=C_{G}(P)$, the element $\rho:=$ $\inf (\eta) \in H^{1}(P, K) \backslash\{0\}$ is $N_{G}(P) / P$-stable. Furthermore $\operatorname{res}_{P, \varphi(P)}(\rho)=0$. Thus, by Lemma 4.1, the image of $\rho$ is a non-zero element in $\mathcal{K}_{\chi}^{1}$. Clearly $\chi$ satisfies condition (b) of Proposition [3.4. Thus an application of Theorem 2.6 finishes the proof.

\section{Remark 5.4.}

(a) There are few examples in the literature where purely group theoretic properties of $G$ allow the computation of the depth of the ring of invariants. The first such instance is the result of Ellingsrud and Skjelbred [4], who showed that (7) holds if $G$ is a cyclic $p$-group. This was extended by Campbell et al. 3] to the case where $G$ is abelian and has a cyclic Sylow $p$-subgroup (see Theorems 1 and 9 in [3]). Corollary 5.3 clearly generalizes this latter result.

(b) A pair $(G, V)$ of a finite group $G$ and a finitely generated $K G$-module $V$ was called "flat" by Kemper [10] if (17) holds, or in our notation here, if $c c_{G}(R)=1$ and $R^{G}$ is flat. Thus we have proved that for a $p$-nilpotent group with cyclic Sylow $p$-subgroup, $(G, V)$ is flat for every representation $V$ in characteristic $p$.

(c) For $p=2$, condition (c) from Theorem 5.3 is always satisfied, since $\mathcal{C}_{G}(P)$ contains $P$ and therefore $\mathcal{N}_{G}(P) / \mathcal{C}_{G}(P)$ has odd order, but on the other hand $\operatorname{Aut}(P)$ is a 2-group since $P$ is a cyclic 2-group. Thus for $p=2$ we can omit the equivalent conditions (a)-(c) in the second statement of Theorem 5.3.

If $G$ is not $p$-nilpotent but $P$ is cyclic, we can use Lemma 4.1 and Proposition 3.4 to obtain the following.

Proposition 5.5. Let $Q:=\varphi(P)$ be the maximal subgroup of $P$ and $m:=c c_{G}(R)$. Assume that there is a non-zero $\rho \in H^{m}(P, R)^{N}$ with $\operatorname{res}_{P, Q}(\rho)=0$. Then $R^{G}$ is flat.

Corollary 5.6. Suppose $W$ is a KP-module such that there exists $\rho \in H^{1}\left(P, W^{*}\right) \backslash$ $\{0\}$ with $\operatorname{res}_{P, Q}(\rho)=0$. Then the invariant ring with respect to the induced module $V:=W^{\uparrow G}$ is flat with:

$$
\operatorname{depth}\left(S\left(V^{*}\right)^{G}\right)=\min \left\{\operatorname{dim}_{K}\left(V^{P}\right)+2, \operatorname{dim}_{K}(V)\right\} .
$$

Proof. Note that $V^{*} \cong W^{* \uparrow G}$, so $\left(W^{* \uparrow N_{G}(P)}\right)_{\mid P}$ is a summand of the module $R_{\mid P}$. Hence the claim follows from Proposition 4.3

Alternatively we can use Proposition 5.5 and the following explicit construction of a suitable element in $H^{1}\left(P, W^{* \uparrow N_{G}(P)}\right)^{N}$ : For each $n \in N$ define $k_{n} \in(\mathbf{Z} / p)^{*}$ by ${ }^{n} \sigma=\sigma^{k_{n}}$. Then $\eta:=\sum_{n \in N} n \otimes k_{n} \cdot \rho \in H^{1}\left(P, W^{* \uparrow N_{G}(P)}\right)^{N}$, which is embedded in $H^{1}\left(P, V^{*}\right)^{N}$. Since $\operatorname{res}_{P, Q}(\eta)=\sum_{n \in N} n \otimes k_{n} \cdot \operatorname{res}_{P, Q}(\rho)=0$, we see from Lemma 4.1 that $m=c c_{G}(R)=1$ and the result follows from Proposition 5.5.

Example 5.7. Let $G$ be the finite simple group $\mathrm{SL}_{2}(8)$ and $p=3$. Then $|G|=$ $7 \cdot 8 \cdot 9$, the Sylow 3 -group $P$ is cyclic with $P \cap{ }^{g} P=1$ for each $g \notin N$ and $N_{G}(P)$ 
is dihedral of order 18. Let $V$ be the permutation module $V:=\left(K_{\downarrow P}\right)^{\uparrow G}$. Then $V_{\downarrow P} \cong K^{(2)} \oplus K[P]^{(6)}$. Hence $\operatorname{dim}_{K}\left(V^{P}\right)=8$ and

$$
\operatorname{depth}\left(S\left(V^{*}\right)^{G}\right)=\min \left\{\operatorname{dim}_{K}\left(V^{P}\right)+2, \operatorname{dim}_{K}(V)\right\}=10<\operatorname{dim}_{K}(V)=56 .
$$

\section{Further APPLicAtions}

The purpose of this section is to provide several examples where $R^{G}$ is flat and $P$ is non-cyclic. Throughout we take $\chi$ to be the set of all proper subgroups of $P$. Thus, by Proposition 3.4, the first condition of Theorem 2.6 is satisfied.

Proposition 6.1. Suppose that $W$ is a P-module and there exists $\rho \in H^{1}(P, W) \backslash$ $\{0\}$ which restricts to zero in $H^{1}(M, W)$ for all maximal subgroups $M \leq P$.

(a) If $W$ is a $P$-module summand of $R$, then $R^{P}$ is flat.

(b) If $W^{\uparrow G}$ is a summand of $R$, then $R^{G}$ is flat and $\operatorname{cc}_{G}(R)=1$.

Proof. Part (a) follows immediately from Theorem 2.6 and part (b) is a consequence of Proposition 4.3 .

Suppose that $W$ is a $P$-module. In order to apply the proposition, we need to identify non-zero elements in $H^{1}(P, W)$ which restrict to zero for all maximal subgroups. Let $N$ and $M$ denote maximal subgroups of $P$. Note that $N$ and $M$ are normal in $P$. The inflation map gives an injection from $H^{1}\left(P / N, W^{N}\right)$ to $H^{1}(P, W)$ (see, for example, [5, Corollary 7.2.3]). Note that $P / N$ is isomorphic to the cyclic group of order $p$ and $W^{N}$ is a vector space over a field of characteristic $p$. Thus, unless $W^{N}$ is a projective $P / N$-module, $H^{1}\left(P / N, W^{N}\right)$ is non-zero. Elements in $H^{1}\left(P / N, W^{N}\right)$ can be represented by vectors in $W^{N}$ which are in the kernel of the transfer (see Theorem 5.1). Assume $W^{N}$ is not projective and choose $u \in W^{N}$ so that the equivalence class, $\{u\}$, is non-zero in $H^{1}\left(P / N, W^{N}\right)$. Clearly the image of $\{u\}$ under the inflation map restricts to zero in $H^{1}(N, W)$. We will formulate a necessary and sufficient condition for the inflation of $\{u\}$ to restrict to zero in $H^{1}(M, W)$. Note that $M /(M \cap N)$ is also isomorphic to the cyclic group of order $p$ so that elements of $H^{1}\left(M /(M \cap N), W^{M \cap N}\right)$ can be represented by vectors in $W^{M \cap N}$.

Lemma 6.2. Suppose that $u \in W^{N}$ represents a non-zero element, $\{u\}$, in $H^{1}\left(P / N, W^{N}\right)$. Then the image of $\{u\}$ in $H^{1}(M, W)$, under the composition of inflation followed by restriction, is zero if and only if $u$ represents zero in $H^{1}\left(M /(M \cap N), W^{M \cap N}\right)$.

Proof. The inflation map is induced by the group epimorphism $P \rightarrow P / N$ and the $K P$-module monomorphism $W^{N} \rightarrow W$. The group epimorphism factors as $P \rightarrow P /(M \cap N) \rightarrow P / N$ while the $K P$-module monomorphism factors as $W^{N} \rightarrow$ $W^{M \cap N} \rightarrow W$. Thus the inflation map factors as

$$
H^{1}\left(P / N, W^{N}\right) \rightarrow H^{1}\left(P /(M \cap N), W^{M \cap N}\right) \rightarrow H^{1}(P, W) .
$$

The composition $H^{1}\left(P /(M \cap N), W^{M \cap N}\right) \rightarrow H^{1}(P, W) \rightarrow H^{1}(M, W)$ is induced by the group homomorphism $M \rightarrow P \rightarrow P /(M \cap N)$ and the $K P$-module monomorphism $W^{M \cap N} \rightarrow W$. The group homomorphism also factors as

$$
M \rightarrow M /(M \cap N) \rightarrow P /(M \cap N)
$$


giving a second factorisation

$$
H^{1}\left(P /(M \cap N), W^{M \cap N}\right) \rightarrow H^{1}\left(M /(M \cap N), W^{M \cap N}\right) \rightarrow H^{1}(M, W) .
$$

Thus the following diagram commutes:

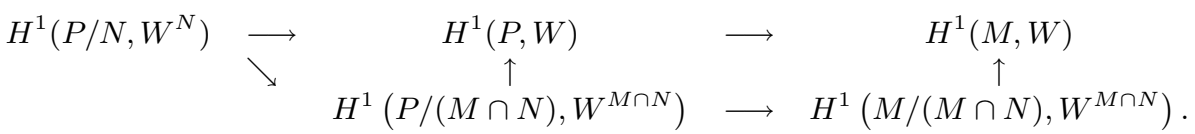

Since the inflation map $H^{1}\left(M /(M \cap N), W^{M \cap N}\right) \rightarrow H^{1}(M, W)$ is injective, the kernel of $H^{1}\left(P / N, W^{N}\right) \rightarrow H^{1}(M, W)$ coincides with the kernel of the map from $H^{1}\left(P / N, W^{N}\right)$ to $H^{1}\left(M /(M \cap N), W^{M \cap N}\right)$. This map takes the element represented by $u$ in $H^{1}\left(P / N, W^{N}\right)$ to the element represented by $u$ in $H^{1}\left(M /(M \cap N), W^{M \cap N}\right)$.

Suppose that $P / P^{\prime}$ is an elementary abelian $p$-group. Choose $\alpha \in P \backslash P^{\prime}$. Then there is a maximal subgroup of $P$, say $N$, such that $P / N=\langle\alpha N\rangle$. Since $P / P^{\prime}$ is an elementary abelian $p$-group, we have $P / P^{\prime}=\left\langle\alpha P^{\prime}\right\rangle \times N / P^{\prime}$. If $M$ is a second maximal subgroup of $P$, then there exists $\gamma \in N$ such that $\alpha \gamma \in M$. Since $M / M \cap N$ is isomorphic to $\mathbf{Z} / p$, we have $M / M \cap N=\langle\alpha \gamma M \cap N\rangle$. Define $\bar{\alpha}:=\alpha P^{\prime}$ and $\bar{\gamma}:=\gamma P^{\prime}$. To apply our technique, we need to find a $u \in W^{N}$ which represents a non-zero class in $H^{1}\left(\langle\bar{\alpha}\rangle, W^{N}\right)$ and represents the zero class in $H^{1}\left(\langle\bar{\alpha} \cdot \bar{\gamma}\rangle, W^{M \cap N}\right)$ for all $M$ and corresponding $\gamma$. If $P / P^{\prime}$ is a rank 2 elementary abelian $p$-group, then $M \cap N=P^{\prime}$ and $\gamma=\beta^{i}$ for a fixed $\beta$ with $i=0, \ldots, p-1$.

Example 6.3. Suppose $P / P^{\prime}$ is a rank 2 elementary abelian $p$-group. Choose $\omega \in \mathbf{F}_{q} \backslash \mathbf{F}_{p}$ and let $U$ be a two-dimensional vector space over $\mathbf{F}_{q}$ with the action of $P / P^{\prime}$ given by

$$
\bar{\alpha}=\left(\begin{array}{cc}
1 & \omega \\
0 & 1
\end{array}\right) \quad \text { and } \quad \bar{\beta}=\left(\begin{array}{ll}
1 & 1 \\
0 & 1
\end{array}\right) .
$$

Take $W^{P^{\prime}}$ to be $S^{\ell}(U)$ with $\ell<p$. Then $W^{P^{\prime}}$ is an $(\ell+1)$-dimensional indecomposable representation of $\left\langle\overline{\alpha \beta^{i}}\right\rangle \cong \mathbf{Z} / p$ and $W^{N}$ is the one-dimensional fixed point space. Thus any non-zero element of $W^{N}$ represents a non-zero class in $H^{1}\left(\langle\bar{\alpha}\rangle, W^{N}\right)$ which maps to zero in $H^{1}\left(\left\langle\overline{\alpha \beta^{i}}\right\rangle, W^{P^{\prime}}\right)$.

Various choices for $P$ and $W$ give rise to this action of $P / P^{\prime}$ on $W^{P^{\prime}}$ :

(i) $W=W^{P^{\prime}}$ and $P^{\prime}=1$. Then $P \cong \mathbf{Z} / p \times \mathbf{Z} / p$. If we take $G=\mathrm{GL}_{2}\left(\mathbf{F}_{p^{2}}\right)$ and $V^{*}=W^{\uparrow G}$, then, by Proposition 6.1 b), $R^{G}$ is flat with $c c_{G}(R)=1$. Thus $\operatorname{dim}\left(R^{G}\right)=(\ell+1)\left(p^{4}-1\right)\left(p^{2}-1\right)$ and $\operatorname{depth}\left(R^{G}\right)=3$.

(ii) Take $\ell=1$ and consider the action on $W$ determined by

$$
\alpha=\left(\begin{array}{ccc}
1 & \omega & 0 \\
0 & 1 & 0 \\
0 & 0 & 1
\end{array}\right) \text { and } \beta=\left(\begin{array}{ccc}
1 & 1 & 0 \\
0 & 1 & 1 \\
0 & 0 & 1
\end{array}\right) .
$$

In this case, as long as $p>2, P \cong(\mathbf{Z} / p \times \mathbf{Z} / p) \rtimes \mathbf{Z} / p$.

(a) Take $V^{*}$ to be the direct sum of $W$ and a two-dimensional vector space on which $\beta$ acts trivially and $\alpha$ acts non-trivially. Then the action of $P$ on $V$ is generated by bireflections, the dimension of $S\left(V^{*}\right)$ is 5 and the depth of $S\left(V^{*}\right)^{P}$ is 4. 
(b) Take $G$ to be $\operatorname{GL}_{3}\left(\mathbf{F}_{p}\right)$ and take $V^{*}=W^{\uparrow G}$. Then by Proposition 6.1 $R^{G}$ is flat with $c c_{G}(R)=1$. Therefore $\operatorname{dim}\left(R^{G}\right)=3\left(p^{3}-1\right)\left(p^{2}-1\right)(p-1)$ and $\operatorname{depth}\left(R^{G}\right)=3$.

Example 6.4. Again suppose $P / P^{\prime}$ is a rank 2 elementary abelian $p$-group. Take $p>2$ and consider the action of $P / P^{\prime}$ on $W^{P^{\prime}}$ given by

$$
\bar{\alpha}=\left(\begin{array}{lll}
1 & 1 & 0 \\
0 & 1 & 1 \\
0 & 0 & 1
\end{array}\right) \quad \text { and } \quad \bar{\beta}=\left(\begin{array}{lll}
1 & 0 & 1 \\
0 & 1 & 0 \\
0 & 0 & 1
\end{array}\right) \text {. }
$$

Then $W^{P^{\prime}}$ is a three-dimensional indecomposable representation of $\left\langle\overline{\alpha \beta^{i}}\right\rangle$ and $W^{N}$ is the one-dimensional fixed point space. Various choices for $P$ and $W$ give rise to this action of $P / P^{\prime}$ on $W^{P^{\prime}}$ :

(i) $W=W^{P^{\prime}}$ and $P^{\prime}=1$. Then $P \cong \mathbf{Z} / p \times \mathbf{Z} / p$.

(a) If we take $G=\mathrm{GL}_{2}\left(\mathbf{F}_{p^{2}}\right)$ and $V^{*}=W^{\uparrow G}$ then, by Proposition 6.1(b), $R^{G}$ is flat with $c c_{G}(R)=1$. Thus $\operatorname{dim}\left(R^{G}\right)=3\left(p^{4}-1\right)\left(p^{2}-1\right)$ and $\operatorname{depth}\left(R^{G}\right)=3$.

(b) Take $V^{*}$ to be the direct sum of $W$ and a two-dimensional vector space on which $\alpha$ acts trivially and $\beta$ acts non-trivially. Then the action of $P$ on $V$ is generated by bireflections, the dimension of $S\left(V^{*}\right)$ is 5 and the depth of $S\left(V^{*}\right)^{P}$ is 4.

(ii) Consider the action on $W$ determined by

$$
\alpha=\left(\begin{array}{llll}
1 & 1 & 0 & 0 \\
0 & 1 & 1 & 0 \\
0 & 0 & 1 & 1 \\
0 & 0 & 0 & 1
\end{array}\right) \text { and } \beta=\left(\begin{array}{llll}
1 & 0 & 1 & 0 \\
0 & 1 & 0 & 0 \\
0 & 0 & 1 & 0 \\
0 & 0 & 0 & 1
\end{array}\right) \text {. }
$$

In this case $P \cong(\mathbf{Z} / p \times \mathbf{Z} / p) \rtimes \mathbf{Z} / p$.

(a) Take $V^{*}$ to be the direct sum of $W$ and a two-dimensional vector space on which $\alpha$ acts trivially and $\beta$ acts non-trivially. Then the dimension of $S\left(V^{*}\right)$ is 6 and the depth of $S\left(V^{*}\right)^{P}$ is 4 .

(b) Take $G$ to be $\mathrm{GL}_{3}\left(\mathbf{F}_{p}\right)$ and take $V^{*}=W^{\uparrow G}$. Then by Proposition 6.1 $R^{G}$ is flat with $c c_{G}(R)=1$. Therefore $\operatorname{dim}\left(R^{G}\right)=4\left(p^{3}-1\right)\left(p^{2}-1\right)(p-1)$ and $\operatorname{depth}\left(R^{G}\right)=3$.

(iii) Consider the action on $W$ determined by

$$
\alpha=\left(\begin{array}{cccc}
1 & 1 & 0 & 0 \\
0 & 1 & 1 & 0 \\
0 & 0 & 1 & 0 \\
0 & 0 & 0 & 1
\end{array}\right) \text { and } \beta=\left(\begin{array}{cccc}
1 & 0 & 1 & 0 \\
0 & 1 & 0 & 0 \\
0 & 0 & 1 & 1 \\
0 & 0 & 0 & 1
\end{array}\right) \text {. }
$$

In this case, if $p>2, P$ is a group of order $p^{4}$. The action of $P$ on $V$ is generated by bireflections, the dimension of $S\left(V^{*}\right)$ is 4 and the depth of $S\left(V^{*}\right)^{P}$ is 3 .

\section{A speCtral SEQUENCE}

The purpose of this section is to prove Theorem 7.1 which is used in the proof of Theorem 2.3. Our proof relies on a spectral sequence analogous to the one used by [4]. For convenience and completeness, we include a development of the spectral sequence.

Let $A$ be a commutative $K$-algebra and $G$ a group acting on $A$ via algebra automorphisms. Then $A$ is a $K G$-module. Furthermore, for each $K G$-module 
$N$ and each $A G$-module $M$, the group $\operatorname{Hom}_{K G}(N, M)$ is an $A^{G}$-module with $(a \cdot \alpha)(x)=a \cdot(\alpha(x))$ for $a \in A^{G}$ and $\alpha \in \operatorname{Hom}_{K G}(N, M)$.

Let $H: K G-\bmod \rightarrow A^{G}-\bmod$ be a contravariant left exact functor. Suppose $S$ is an $A^{G}$-module and $N$ is a $K G$-module. We choose a projective resolution of $S$ as an $A^{G}$-module,

$$
P^{*}=\cdots P^{2} \rightarrow P^{1} \rightarrow P^{0} \rightarrow S \rightarrow 0,
$$

and a free resolution of $N$ as a $K G$-module,

$$
B^{*}=\cdots B^{2} \rightarrow B^{1} \rightarrow B^{0} \rightarrow N \rightarrow 0 .
$$

Then we obtain a canonical first quadrant double complex

$$
F^{i, j}:=\operatorname{Hom}_{A^{G}}\left(P^{i}, H\left(B^{j}\right)\right) .
$$

Attached to this double complex are two spectral sequences. The first is constructed using the 'horizontal filtration' and satisfies

$$
{ }_{h o r} \mathrm{E}_{1}^{p, q}=H^{q}\left(\operatorname{Hom}_{A^{G}}\left(P^{*}, H\left(B^{p}\right)\right)\right) \cong \operatorname{Ext}_{A^{G}}^{q}\left(S, H\left(B^{p}\right)\right),
$$

while the second is constructed using the 'vertical filtration' and satisfies

$$
{ }_{v e r} \mathrm{E}_{1}^{p, q}=H^{q}\left(\operatorname{Hom}_{A^{G}}\left(P^{p}, H\left(B^{*}\right)\right)\right) \cong \operatorname{Hom}_{A^{G}}\left(P^{p}, \mathcal{R}^{q} H(N)\right),
$$

where $\mathcal{R}^{q} H$ denotes the $q$-th right derived functor of $H$. It is now straightforward to see that

$$
{ }_{v e r} \mathrm{E}_{2}^{p, q} \cong \operatorname{Ext}_{A^{G}}^{p}\left(S, \mathcal{R}^{q} H(N)\right) .
$$

From elementary properties of first quadrant double complexes, it is clear that both spectral sequences converge to the graded cohomology of the total complex, where the gradings are defined using the horizontal or vertical filtration, respectively. Furthermore, for each of these spectral sequences the $r^{\text {th }}$ differential has bidegree $(r, 1-r)$.

We want to apply this in the situation where $S:=A^{G} / I$ for an ideal $I \unlhd A^{G}$, $N \cong K$, the trivial $K G$-module, and $H(X):=\operatorname{Hom}_{K G}(X, A)$, the contravariant Hom-functor. Thus $H\left(B^{j}\right)=\operatorname{Hom}_{K G}\left(B^{j}, A\right) \cong A^{m_{j}}$, where $m_{j}$ is the rank of the free $K G$-module $B^{j}$. Therefore

$$
\begin{gathered}
{ }_{h o r} \mathrm{E}_{1}^{p, q} \cong \operatorname{Ext}_{A^{G}}^{q}\left(A^{G} / I, A^{m_{p}}\right) ; \\
{ }_{v e r} \mathrm{E}_{2}^{p, q} \cong \operatorname{Ext}_{A^{G}}^{p}\left(A^{G} / I, \operatorname{Ext}_{K G}^{q}(K, A)\right) ; \\
v e r \mathrm{E}_{2}^{p, 0} \cong \operatorname{Ext}_{A^{G}}^{p}\left(A^{G} / I, A^{G}\right) .
\end{gathered}
$$

Assume that $h:=\operatorname{grade}(I, A)$ is finite. (For example, if $A$ is a Cohen-Macaulay ring, and $G$ is finite, then $h$ is the height of $I$.) Then for all $q<h$ we have ${ }_{h o r} \mathrm{E}_{1}^{p, q}=0$ and hence ${ }_{h o r} \mathrm{E}_{\infty}^{p, q}=0$. Using the horizontal filtration $\mathcal{F}$ of the total complex $T:=\operatorname{tot}\left(F^{i, j}\right)$ and the convergence

$$
{ }_{h o r} \mathrm{E}_{r}^{p, q} \Rightarrow{ }_{h o r} \mathrm{E}_{\infty}^{p, q} \cong \mathcal{F}^{p}\left(H^{p+q}(T)\right) / \mathcal{F}^{p+1}\left(H^{p+q}(T)\right)
$$

we get for every $n=p+q<h$ :

$$
0=\mathcal{F}^{n+1}\left(H^{n}(T)\right)=\mathcal{F}^{n}\left(H^{n}(T)\right)=\mathcal{F}^{n-1}\left(H^{n}(T)\right)=\cdots=\mathcal{F}^{0}\left(H^{n}(T)\right)=H^{n}(T) .
$$

Therefore ${ }_{v e r} E_{\infty}^{p, q}=0$ for $p+q<h$. 
Theorem 7.1. Let $m$ denote the cohomological connectivity $c_{G}(R)$. Suppose that $I$ is a prime ideal in $R^{G}$ and $m+1$ is less than the height, $h$, of $I$. Then $\operatorname{grade}\left(I, H^{m}(G, R)\right)=0$ if and only if $\operatorname{grade}\left(I, R^{G}\right)=m+1$.

Proof. We use the spectral sequence ${ }_{v e r} \mathrm{E}_{*}^{p, q}$ from the preceding discussion. Since $R$ is Cohen-Macaulay, grade $(I, R)=h$. Therefore ${ }_{v e r} \mathrm{E}_{\infty}^{p, q}=0$ for $p+q<h$. In particular, ${ }_{v e r} \mathrm{E}_{\infty}^{0, m}=0$. Furthermore, since $H^{i}(G, R)=0$ for $0<i<m$, we have ${ }_{v e r} \mathrm{E}_{2}^{p, q}=0$ for $0<q<m$.

Suppose $\operatorname{grade}\left(I, H^{m}(G, R)\right)=0$. Then

$$
{ }_{v e r} \mathrm{E}_{2}^{0, m}=\operatorname{Ext}_{R^{G}}^{0}\left(R^{G} / I, H^{m}(G, R)\right) \neq 0 .
$$

Suppose $\alpha \in{ }_{\text {ver }} \mathrm{E}_{2}^{0, m} \backslash\{0\}$. Since ${ }_{\text {ver }} \mathrm{E}_{\infty}^{0, m}=0$ and the spectral sequence is first quadrant, there is a differential $d_{r}$ such that $d_{r}(\alpha) \neq 0$. However, ${ }_{\text {ver }} E_{2}^{p, q}=0$ for $0<q<m$. Thus $r=m+1$ and, since $d_{m}$ has bidegree $(m, 1-m)$, we conclude that ${ }_{\text {ver }} \mathrm{E}_{2}^{m+1,0} \neq 0$. Furthermore, $\operatorname{grade}\left(I, R^{G}\right)$ is the smallest $p$ such that ${ }_{\text {ver }} E_{2}^{p, 0} \neq 0$. Thus grade $\left(I, R^{G}\right) \leq m+1$. However any non-zero element of ver $E_{2}^{p, 0}$ for $p<m+1$ would survive the spectral sequence giving a non-zero element in ${ }_{v e r} E_{\infty}^{p, 0}$. Therefore $\operatorname{grade}\left(I, R^{G}\right)=m+1$.

Suppose $\operatorname{grade}\left(I, H^{m}(G, R)\right)>0$. Then

$$
{ }_{v e r} \mathrm{E}_{2}^{0, m}=\operatorname{Ext}_{R^{G}}^{0}\left(R^{G} / I, H^{m}(G, R)\right)=0 .
$$

Thus any non-zero element of ${ }_{v e r} E_{2}^{p, 0}$ for $p \leq m+1<h$ would survive the spectral sequence giving a non-zero element in ${ }_{v e r} E_{\infty}^{p, 0}$. Thus grade $\left(I, R^{G}\right)>m+1$.

\section{REFERENCES}

[1] David J. Benson, Representations and Cohomology I, Cambridge Studies in Advanced Mathematics 30, Cambridge Univ. Press, Cambridge 1991. MR92m:20005

[2] Winfried Bruns, Jürgen Herzog, Cohen-Macaulay Rings, Cambridge University Press, Cambridge 1993. MR:95h:13020

[3] H. E. A. Campbell, I. P. Hughes, G. Kemper, R. J. Shank, D. L. Wehlau, Depth of Modular Invariant Rings, Transformation Groups 5 (2000), 21-34. MR2001a:13004

[4] Geir Ellingsrud, Tor Skjelbred, Profondeur d'anneaux d'invariants en caractéristique $p$, Compos. Math. 41 (1980), 233-244. MR82c:13015

[5] Leonard Evens, The Cohomology of Groups, Oxford University Press, Oxford 1991. MR 93i:20059

[6] Peter Fleischmann, Relative Trace Ideals and Cohen-Macaulay Quotients of Modular Invariant Rings, P. Dräxler, G.O. Michler, C. M. Ringel, eds., Computational Methods for Representations of Groups and Algebras, Euroconference in Essen, April 1-5 1997, Progress in Mathematics 173, Birkhäuser, Basel 1999. MR2000j:13007

[7] Peter Fleischmann, R. James Shank, The Relative Trace Ideal and the Depth of Modular Rings of Invariants, Arch. der Math. 80 (2003), 347-353.

[8] Bertram Huppert, Endliche Gruppen I, Springer-Verlag, Berlin, Heidelberg, New York 1967. MR:37:302

[9] Gregor Kemper, On the Cohen-Macaulay Property of Modular Invariant Rings, J. of Algebra 215 (1999), 330-351. -MR2000d:13008

[10] Gregor Kemper, The Depth of Invariant Rings and Cohomology, with an appendix by Kay Magaard, J. of Algebra 245 (2001), 463-531. MR2002h:13009

[11] Peter Landrock, Finite Group Algebras and their Modules, Lond. Math. Soc. Lecture Note Ser. 84, Cambridge Univ. Press, Cambridge 1983. MR85h:20002 
[12] Martin Lorenz, Jay Pathak, On Cohen-Macaulay Rings of Invariants, J. of Algebra 245 (2001), 247-264. MR2002h:13010

[13] Larry Smith, Polynomial Invariants of Finite Groups, A. K. Peters, Wellesley, Mass. 1995. MR 96f: 13008

Institute of Mathematics and Statistics, University of Kent, Canterbury, CT2 7NF, UNITED KINGDOM

E-mail address: P.Fleischmann@kent.ac.uk

Zentrum Mathematik - M11, Technische Universität München, Boltzmannstr. 3, 85748 Garching, Germany

E-mail address: kemper@ma.tum.de

Institute of Mathematics and Statistics, University of Kent, Canterbury, CT2 7NF, United KingDOM

E-mail address: R.J.Shank@kent.ac.uk 